\title{
RAPID DETERMINATION OF FOOD QUALITY USING STEADY STATE FREE PRECESSION SEQUENCES IN TD-NMR SPECTROSCOPY
}

\author{
L.A. Colnago ${ }^{1}$, T.B. Moraes ${ }^{2}$, T. Monaretto ${ }^{3}$, F.D. Andrade $^{1}$ \\ ${ }^{1}$ Embrapa Instrumentação, Rua XV de Novembro 1452, São Carlos-SP, 13560-970, Brazil. \\ ${ }^{2}$ Instituto de Física de São Carlos, Universidade de São Paulo, Avenida Trabalhador São- \\ Carlense 400, São Carlos-SP, 13566-590, Brazil. \\ ${ }^{3}$ Instituto de Química de São Carlos, Universidade de São Paulo, Avenida Trabalhador São- \\ Carlense 400, São Carlos-SP, 13566-590, Brazil.
}

\section{INTRODUCTION}

The use of time-domain NMR spectroscopy (TD-NMR) in food science began more than 40 years ago with the introduction of the small benchtop NMR analyzer. ${ }^{1}$ Since then, TD-NMR has become one of the most robust, rapid, cost-effective and versatile tools in the food industry. Earlier TD-NMR applications were primarily based on quantitative analysis using the intensity of free induction decay (FID) and/or spin echo signals. ${ }^{1-3}$ In the last two decades, the use of relaxometry and/or diffusometry methods have expanded the application TD-NMR in food science exponentially. ${ }^{2,3}$

The majority of these applications use the Carr-Purcell-Meiboom-Gill (CPMG) pulse sequence. ${ }^{1-3}$ This sequence is very robust ${ }^{4}$, rapid and yields an exponential decay that is dependent upon the transverse relaxation time $\left(\mathrm{T}_{2}\right) .{ }^{1-3}$ Therefore, CPMG has been used as an all-purpose sequence in TD-NMR applications and is a standard pulse sequence present in commercial and homemade TD-NMR spectrometers. CPMG has been used to study food products such as oilseeds, fresh meat, fish, and fruit, as well as industrialized and packaged food products. ${ }^{1,5,6}$

The longitudinal relaxation time $\left(T_{1}\right)$ measurements using inversion-recovery (IR) or progressive saturation pulse sequences have rarely been used in food analysis due to the length of experiment time. ${ }^{2,6}$ Pulsed field gradient spin-echo (PFGSE) pulse sequences are the second most used pulse sequence in TD-NMR applications. ${ }^{2}$ PFGSE has been used to measure the water self-diffusion coefficient, water mobility, and droplet size in several food products. However, PFGSE requires an additional hardware accessory that is not available for all TD-NMR spectrometers. Thus, there is an effort towards the development and implementation of rapid TD-NMR analytical methods that meet the growing demand for tools of quality assessment.

Accordingly, we have been developing steady-state free precession (SSFP) pulse sequences for TD-NMR spectroscopy since $2000 .^{7}$ SSFP sequences have been used in quantitative analysis similarly to analyses performed with FID or spin echo. ${ }^{7,8}$ However, the signal-tonoise ratio (SNR) with SSFP is much higher than that obtained with FID or echo in the same 
average time. ${ }^{7,8}$ Moreover, SSFP sequences can also be used in fast flow (online) quantitative measurements of liquid or solid samples. ${ }^{9,10}$ The theory for quantitative analysis using the amplitude of an SSFP signal is presented in section 2.1.

Further advantages of SSFP sequences are: the dependence of the transient signals on two relaxation times $\left(T_{1}\right.$ and $\left.T_{2}\right)$, the data are collected in a length of time similar to CPMG and it does not require special hardware and therefore can be implemented on any modern TDNMR spectrometer. ${ }^{1,6,8,11}$ The theory for the evolution of the NMR signal submitted to a train of pulses (SSFP sequence) is presented in section 2.2.

\section{THEORY}

\subsection{Amplitude of the NMR signal in the SSFP regime}

SSFP sequences have been used to improve the SNR in pulsed NMR spectroscopy since $1958 .^{12}$ It is a simple pulse sequence consisting of a train of radiofrequency pulses $(r f)$ with the same phase and flip angle $(\theta)$, and the time between pulses $\left(T_{p}\right)$ is shorter than $T_{2}\left(T_{p}<\right.$ $\mathrm{T}_{2}$ ) (Figure 1).

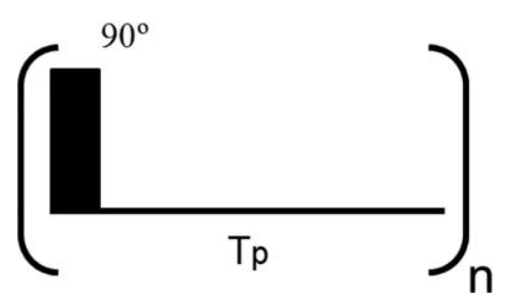

Figure 1 Diagram of the SSFP pulse sequence, where $n$ is number of rf pulses.

In 1966, Ernst and Anderson derived the analytical solution for the SSFP regime. ${ }^{13}$ They showed that the SSFP signal is composed of FID and echo signals. The echo component (M-) immediately preceding the pulse is given by equations 1 through 3 , and the FID $(\mathrm{M}+)$ component is given by equations 4 through 6 .

$$
\begin{aligned}
& M_{x}^{-}=\frac{M_{0}\left(1-E_{1}\right)\left[E_{2} \sin \theta \sin \Phi\right]}{D} \\
& M_{y}^{-}=\frac{M_{0}\left(1-E_{1}\right)\left[E_{2} \sin \theta \cos \Phi-E_{2}^{2} \sin \theta\right]}{D} \\
& M_{z}^{-}=\frac{M_{0}\left(1-E_{1}\right)\left[1-E_{2} \cos \Phi-E_{2} \cos \theta\left(\cos \theta-E_{2}\right)\right]}{D} \\
& M_{x}^{+}=M_{x}^{-} \\
& M_{y}^{+}=\frac{M_{0}\left(1-E_{1}\right)\left[\left(1-E_{2} \cos \Phi\right) \sin \theta\right]}{D} \\
& M_{z}^{+}=\frac{M_{0}\left(1-E_{1}\right)\left[E_{2}\left(E_{2} \cos \Phi\right)+\left(1-E_{2} \cos \Phi\right) \cos \theta\right]}{D}
\end{aligned}
$$


where $D=\left[\left(1-E_{1} \cos \theta\right)\left(1-E_{2} \cos \Phi\right)\right]-\left[\left(E_{1}-\cos \theta\right)\left(E_{2}-\cos \Phi\right) E_{2}\right]$, with the precession angle $\Phi=\Omega t$, offset frequency $\Omega=\varpi_{\text {ref }}-\varpi_{0}$, and relaxation components $E_{1}=\exp \left(-T_{p} / T_{1}\right)$ and $E_{2}=\exp \left(-T_{p} / T_{2}\right)$.

With these equations it is possible to calculate the magnitude of the magnetization in the $x y$ plane after the $\mathrm{n}^{\text {th }} \mathrm{rf}$ pulse, assuming $\mathrm{T}_{\mathrm{p}}<<\mathrm{T}_{1} \cdot{ }^{14}$

$$
|\vec{M}|=\frac{M_{0}|\sin (\theta)| \sqrt{2-2 \cos \Phi}}{(1+\cos \theta)(1-\cos \Phi)+(1-\cos \theta) 2 T_{1} / T_{2}}
$$

Therefore, the amplitude of the SSFP signal is dependent upon the flip angle $\theta$, precession angle $\Phi=\Omega T_{p}$ and $\mathrm{T}_{1} / \mathrm{T}_{2}$ ratio. ${ }^{7,14}$ The magnetization goes to null $|M| \rightarrow 0$, when $\sqrt{2-2 \cos \Phi} \rightarrow 0$ or

$$
\Phi=n 2 \pi
$$

where $n$ is an integer.

Figure 2 shows the dependence of the NMR signal amplitude upon the precession angle $\Phi$ and frequency offset for $\theta=45^{\circ}$ and $90^{\circ}, \mathrm{T}_{\mathrm{p}}=0.3 \mathrm{~ms}, \mathrm{~T}_{1}=150 \mathrm{~ms}$ and $\mathrm{T}_{2}=50 \mathrm{~ms}$, according to equation 7 .

For $\Phi=n 2 \pi$, the magnetization is minimal because the FID and echo components are dephased by $180^{\circ}$, resulting in destructive interference. For $\Phi=(2 n+1) \pi$, the FID and echo are in phase and the constructive interaction creates a maximum signal intensity when $\theta=$ $90^{\circ}$.

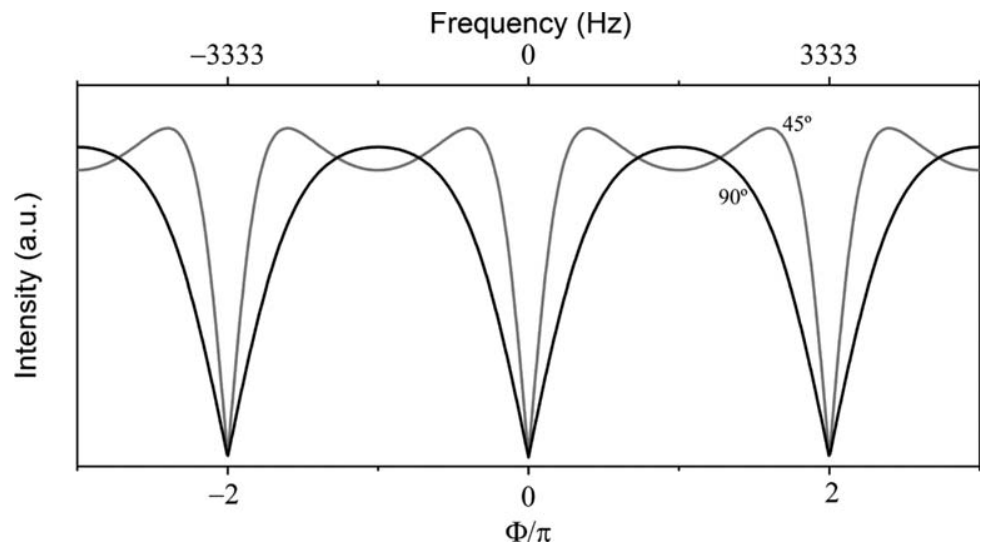

Figure 2 Dependence of the normalized SSFP signal amplitude upon the precession angle $\Phi$ and frequency offset when $\theta=45^{\circ}$ and $90^{\circ}, T_{p}=0.3 \mathrm{~ms}, T_{1}=150 \mathrm{~ms}$ and $T_{2}=50 \mathrm{~ms}$.

Equations 1 through 6 show that the behavior of the magnetization in the SSFP regime is complex and depends on a series of experimental parameters, such as $\Phi, \theta, \Omega, T_{p}, T_{1}$ and $T_{2}$. However, these analytical descriptions do not include the effect of other parameters on the SSFP signal, such as $\mathrm{T}_{\mathrm{p}}$ variation and phase alternation.

To fully describe the SSFP phenomenon we have numerically simulated (Matlab) ${ }^{15}$ the 
influence of the all the above parameters based on the rotation matrix and the method of the sum of isocromats ${ }^{16}$, in which the Lorentzian distribution was assumed ${ }^{9}$.

$$
g(\varpi 0)=\frac{\left(T_{2}^{*} / \pi\right)}{1+\left(\varpi-\varpi_{0}\right)^{2} T_{2}^{* 2}}
$$

and

$$
\frac{1}{T_{2}^{*}}=\frac{1}{T_{2}}+\frac{1}{T_{2 \text { inom }}}
$$

where $T_{\text {2inom }}=1 / \gamma \Delta B_{0}$.

Figure 3 shows the numerical simulations for the TD-NMR signals after reaching the steady state regime. The time necessary to reach the steady state is discussed in section 2.2. The steady state signals were simulated using $\mathrm{T}_{1}=100 \mathrm{~ms}, \mathrm{~T}_{2}=50 \mathrm{~ms}, \mathrm{~T}_{2} *=0.5 \mathrm{~ms}, \theta=90^{\circ}$, a frequency offset of 8.333 (Figures $3 \mathrm{~A}$ to $\mathrm{D}$ ) and 6.666 (Figure $3 \mathrm{E}$ ) $\mathrm{KHz}$ and various $\mathrm{T}_{\mathrm{p}}$ values. In Figure 3, the pulse is observed in the center of the window $(t=0)$. The FID component after the pulse is on the right side of $t=0$, and the echo component is on the left side of the pulse.

Figure 3A shows the NMR signal for $T_{p}=5 T_{1}$. This figure shows an FID signal with maximum amplitude. With this pulse repetition rate, the echo signals are not observed.

Figure $3 \mathrm{~B}$ shows the NMR signal for $\mathrm{T}_{\mathrm{p}}=\mathrm{T}_{2}$. In this condition the NMR signal is in the SSFP regime and is composed of an FID and an echo between the pulses. The FID signal has higher amplitude than the echo signal. The FID amplitude in Figure 3B is lower than that of the FID in Figure $3 \mathrm{~A}$ because $\mathrm{T}_{\mathrm{p}}<5 \mathrm{~T}_{1}$, which does not allow the return of the magnetization to thermal equilibrium.

Figures $3 \mathrm{C}$ to $\mathrm{E}$ depicts more than one period between the pulses, in the interval of -1.5 to 1.5 ms. Figure $3 \mathrm{C}$ shows two periods for $\mathrm{T}_{\mathrm{p}}=2.9 \mathrm{~T}_{2} *$ in which the FID and echo signals have similar amplitudes, and the FID decays faster than $\mathrm{T}_{2} *$ compared to the FID decay in Figures $3 \mathrm{~A}$ and $\mathrm{B}$. This faster decay is due to the partial destructive interaction between the FID and echo in the center of the SSFP signals.

Figures $3 \mathrm{D}$ and $\mathrm{E}$ show the SSFP signals for $\mathrm{T}_{\mathrm{p}}=0.3 \mathrm{~ms}<\mathrm{T}_{2} *$. In this condition the overlap between the FID and the echo signal is maximal, yielding a special SSFP regime, known as Continuous Wave Free Precession (CWFP). ${ }^{6,10}$ The amplitude of CWFP signal is strongly dependent upon $\Phi=\Omega T_{p}$, as shown in Figure 2. Figure 3D depicts the maximum CWFP signal when $\Phi=5 \pi$ with a frequency offset of $8.333 \mathrm{KHz}$ (constructive interference) and Figure $3 \mathrm{E}$ depicts the minimal CWFP signal when $\Phi=4 \pi$ with a frequency offset of 6.666 $\mathrm{KHz}$ (destructive interaction).

According to equation 11, the magnitude of the CWFP signal when $\theta=90^{\circ}$ and $\Phi=5 \pi$ is dependent upon the $T_{1} / T_{2}$ ratio. ${ }^{1,6,11}$

$$
\left|M_{s s}\right|=\frac{M_{0}}{1+\frac{T_{1}}{T_{2}}}
$$




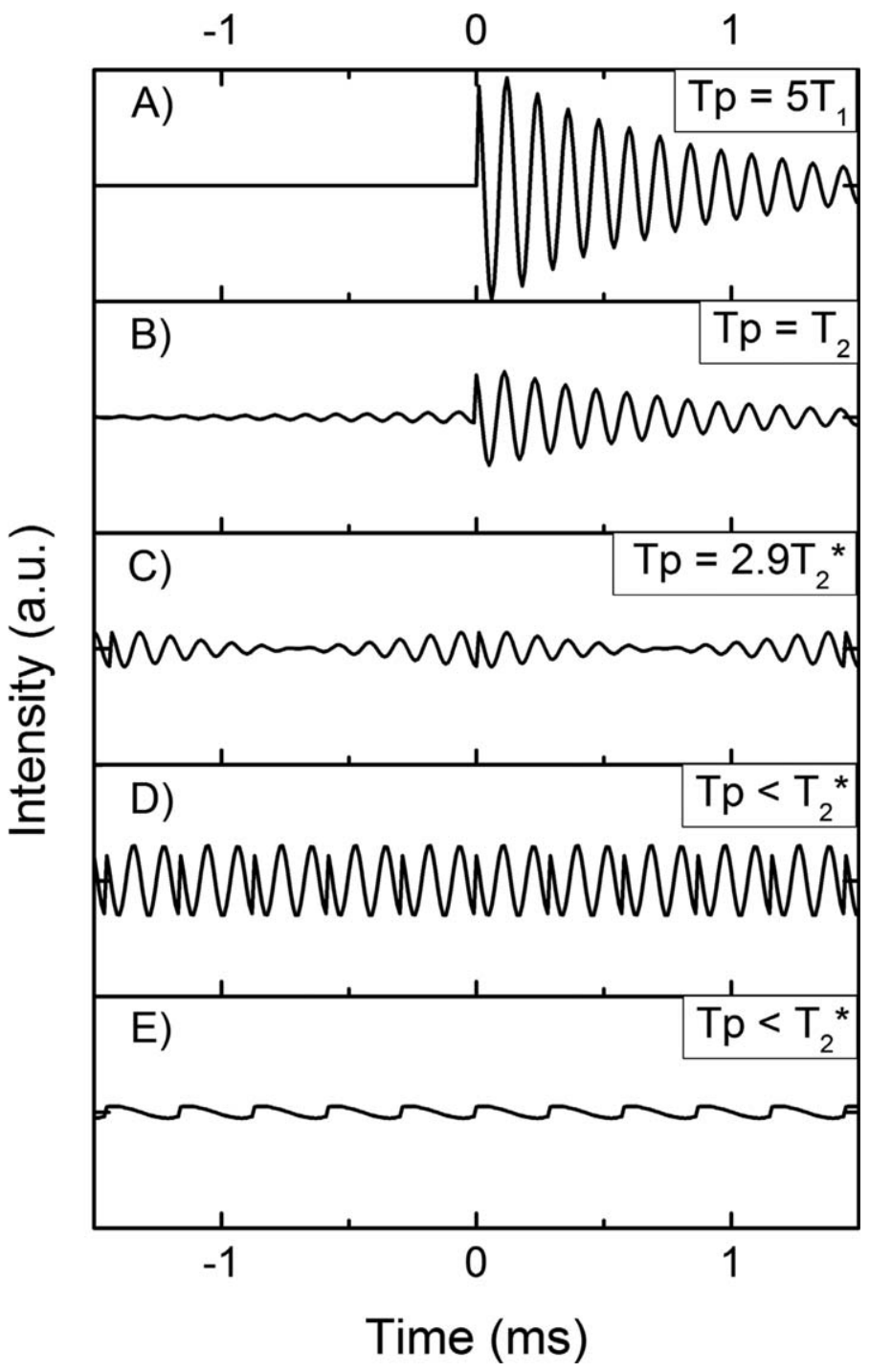

Figure 3 NMR signals simulated numerically using $T_{1}=150 \mathrm{~ms}, T_{2}=50 \mathrm{~ms}, T_{2} *=0.5 \mathrm{~ms}$ and several $T_{p}$ values. A) $\left.\left.\left.T_{p}=5 T_{1}, B\right) T_{p}=T_{2}, C\right) T_{p}=2.9 T_{2}{ }^{*}, D\right)$ and E) $T_{p}<T_{2}{ }^{*}$. The frequency offset is $8.333 \mathrm{KHz}$ (A to D) and $6.666 \mathrm{KHz}(E)$.

The magnitude of the CWFP signal is not dependent on the pulse repetition rate, as in conventional pulse sequences (Figure $3 \mathrm{~A}$ and $\mathrm{B}$ ). Instead, it depends on the $T_{1} / T_{2}$ ratio (equation 11), and the repetition time can be short $\left(\mathrm{T}_{\mathrm{p}}<<\mathrm{T}_{1}, \mathrm{~T}_{2}<\mathrm{T}_{2}{ }^{*}\right)$ and without saturation (Figure 3D). Therefore, thousands of CWFP signals can be averaged during one $\mathrm{T}_{1}$ period, thereby enhancing the SNR by one order of magnitude in the same average time used for FID or echo signals. ${ }^{7,8}$ The magnitude of CWFP signals has been used in quantitative analysis, in conventional benchtop spectrometers and in online measurements using long Halbach and superconducting magnets ${ }^{1,6,9}$. 


\subsection{Transient SSFP signal}

The evolution of the NMR signal, submitted to a train of pulses (SSFP sequence) has been used in several applications in TD-NMR. In 1977, Kronenbitter and Schwenk proposed the use of the transient SSFP signal to measure $\mathrm{T}_{1}$ and $\mathrm{T}_{2}{ }^{17}$ The method consists of two steps: First, the measurements of the $T_{1} / T_{2}$ ratio, by measuring the maximum amplitude of the SSFP signal as a function of the flip angle $(\theta)$ to obtain the optimum $\theta\left(\theta_{\text {opt }}\right)$, and second, the use of $\theta_{\text {opt }}$ to measure the time constant $\left(T^{*}\right)$, equation 12 , for the evolution of the SSFP signal, yielding the $T_{1}+T_{2}$ value. With these two measurements it is possible to determine both relaxation times. $^{17}$

$$
T^{*}=\frac{2 T_{1} T_{2}}{T_{1}(1-\cos \theta)+T_{2}(1+\cos \theta)}
$$

In 2006, Venâncio T. et al reported that is not necessary to use two steps to measure both relaxation times when the transient SSFP signal is obtained with $\theta=90^{\circ}$ and $\Phi=(2 n+1) \pi^{11}$

Figure 4 shows the evolution of the magnitude of the CWFP signal from the first pulse to the stationary regime $\left(\left|\mathrm{M}_{\mathrm{s} S}\right|\right)$. This signal undergoes two transient regimes before the steady state is reached. The first transient regime (dark grey) shows an alternation of the amplitude between even and odd pulses followed by signal decay, with the time constant of $\mathrm{T}_{2}{ }^{*}$. When the alternations subside, the signal reaches a quasi-stationary state (light grey). The decay of the quasi-stationary state to the stationary state (white) is governed by the time constant $\mathrm{T}^{*}$. For $\theta=90^{\circ}$, equation 12 is reduced to:

$$
T^{*}=\frac{2 T_{1} T_{2}}{T_{1}+T_{2}}
$$

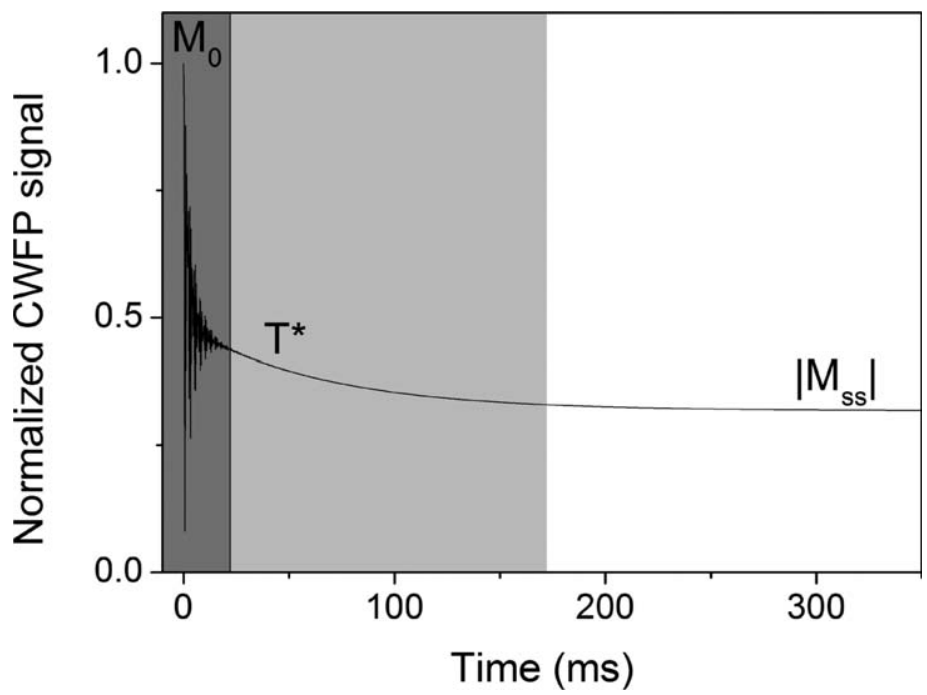

Figure 4 Evolution of the CWFP signal magnitude from the first pulse to the stationary state $\left(M_{s s}\right)$. 
Upon rewriting equations 11 and 13, we obtain the following:

$$
T_{1}=\frac{T^{*} / 2}{\left|M_{s s}\right| / M_{0}}
$$

and

$$
T_{2}=\frac{T^{*} / 2}{1-\left|M_{s s}\right| / M_{0}}
$$

Therefore, upon measuring the magnitude of the signal after the first pulse $\left|M_{0}\right|$, the magnitude of the CWFP signal $\left|M_{s s}\right|$ and $\mathrm{T}^{*}$, it is possible to calculate the relaxation times in a single scan experiment using equation $14 .{ }^{11}$ The $\mathrm{T}^{*}$ value is calculated by fitting the $\mathrm{T}^{*}$ decay with an exponential decay function. The $T_{1}$ and $T_{2}$ values are obtained with single CWFP experiments are similar to those obtained by Inversion recovery $\left(\mathrm{T}_{1}\right)$ and CPMG $\left(\mathrm{T}_{2}\right)$ pulse sequences. $^{11}$

When $T_{1} \sim T_{2}$ there is only a small difference in amplitude between the quasi-stationary state and the stationary state of the CWFP signal. This might yields, a $T^{*}$ with large error when the CWFP signal has low SNR. ${ }^{4}$

To solve this problem we proposed the use of a Carr-Purcell sequence, using $90^{\circ}$-refocusing pulses, also known as CP-CWFP (Figure 5). ${ }^{4}$ The only difference between CWFP and CPCWFP sequences is the addition of a pulse, which separates the CWFP pulse train (Figure 1) by the time interval $T_{p} / 2$. The effect of this modification is shown in Figure 6 . The CP-CWFP signal intensity decays to a minimum value (quasi-stationary state) and then increases to the same amplitude observed in the CWFP regime. As shown in Figure 6A, the amplitude variation during $\mathrm{T}^{*}$ is much more pronounced in CP-CWFP than in the CWFP sequence. This results in improved fitting of $T^{*}$ for a sample when $T_{1} \sim T_{2}$.

Conversely, CP-CWFP signals yield a small difference in amplitude during $\mathrm{T}^{*}$ when $\mathrm{T}_{1} \gg$ $\mathrm{T}_{2}$ (Figure $6 \mathrm{~B}$ ). In this case, $\mathrm{T}^{*}$ of CWFP can be fitted with minimal error. When $\mathrm{T}_{1}>\mathrm{T}_{2}$ (Figure 6C) CWFP and CP-CWFP have similar amplitude variations during $\mathrm{T}^{*}$.

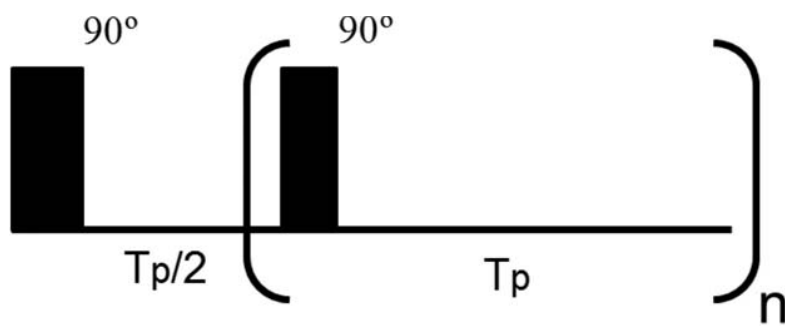

Figure 5 Diagram of $C P-C W F P$ pulse sequence.

In addition to measuring the relaxation times, the CWFP or CP-CWFP signals have been used to obtain qualitative and quantitative information from food products using uni- and multivariate analyses. ${ }^{1,5,18-20}$

The ratio $\left|\mathrm{M}_{\mathrm{ss}}\right| \mathrm{M}_{0}$ of the CWFP signal has a higher correlation with intramuscular fat content and water loss during cooking (cooking loss) in beef than $T_{2}$ measured by CPMG. ${ }^{18,19}$ $\left|\mathrm{M}_{\mathrm{ss}}\right| \mathrm{M}_{0}$ also has a higher univariate correlation with animal sex and genetics than CPMG. ${ }^{20}$ However, the multivariate analysis results in similar beef classification (sex and genetics) using the full CPMG and CWFP data sets. ${ }^{20}$ 

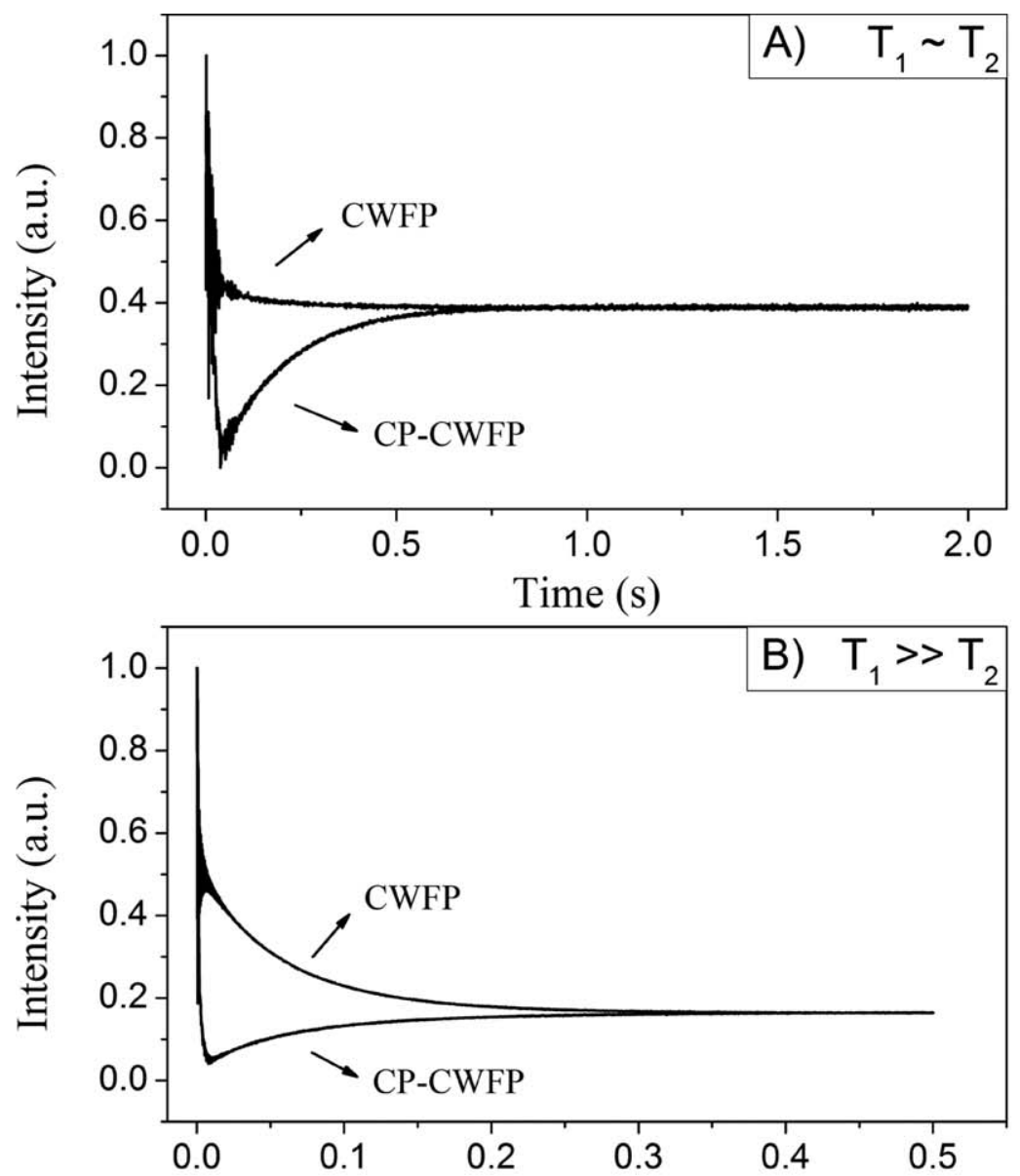

Time (s)

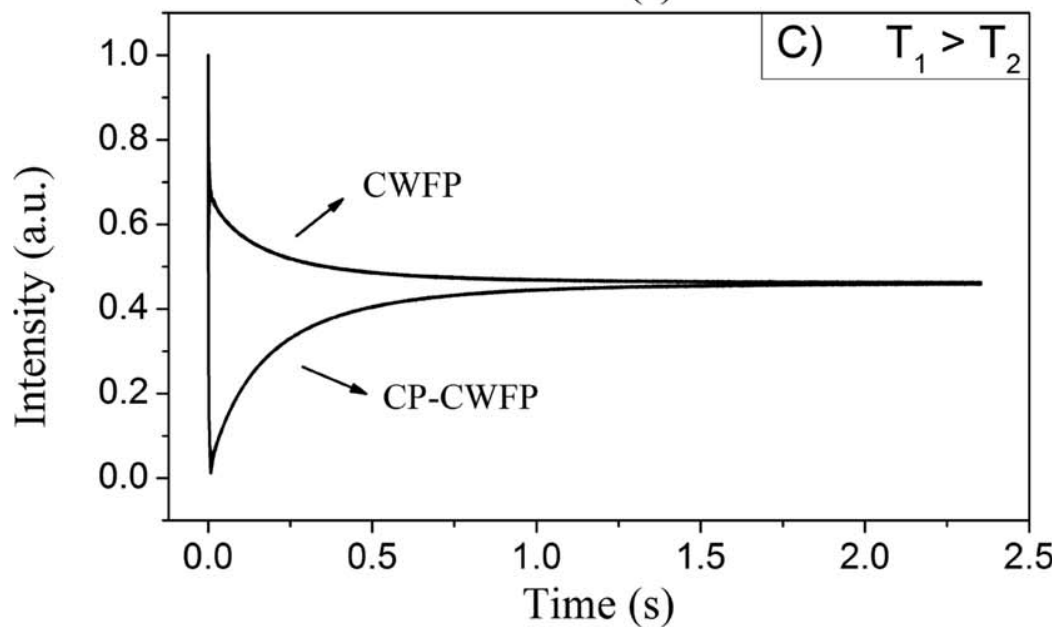

Figure 6 Experimental $C W F P$ and $C P$-CWFP signal intensities for tap water (A), hotdog sausage (B) and mayonnaise $(C)$ samples. 
CWFP and CP-CWFP sequences are a useful alternative to CPMG because the analysis can be performed in the same amount time, they do not require special hardware and, therefore, the sequences can be implemented in any modern TD-NMR spectrometer. The scripts for the CWFP and CP-CWFP sequences for the Minispec spectrometer (BRUKER) are described in the appendix I.

\section{COMPARISON BETWEEN CPMG, CWFP AND CP-CWFP ANALYSES OF FRESH AND PROCESSED FOOD PRODUCTS}

To demonstrate the potential of CWFP and CP-CWFP analyses in food science we compared them with CPMG, using a benchtop spectrometer, SLK 100, Spinlock (Córdoba, Argentine).

Figure 7 shows the CPMG, CWFP and CP-CWFP signals for ripe and unripe grapes and bananas. Figures 7A and B show the CPMG and CWFP/CP-CWFP signals of ripe (brix $=18$ ) and unripe (brix $=10$ ) grapes. The CPMG decays for both grapes were similar. However, the ripe grapes, with a high sugar content, shows a more rapid CPMG decay $(1.0 \mathrm{~s})$ than the unripe grapes $(1.2 \mathrm{~s})$, with low sugar content, as expected. ${ }^{21}$ Similar results for ripe and unripe grapes were also observed for CWFP and CP-CWFP signals (7B). The small and large variations in the amplitude during $\mathrm{T}^{*}$ for CWFP and CP-CWFP, respectively, and similar $\left|\mathrm{M}_{\mathrm{ss}}\right|$ amplitudes (0.37 and 0.4, respectively) indicate that $\mathrm{T}_{1} \sim \mathrm{T}_{2}$ for both grapes.

Conversely, the CPMG, CWFP and CP-CWFP signals (Figures 7C and D) of ripe and unripe bananas are much more distinct. The decay of the CPMG signal $(7 \mathrm{C})$ of unripe bananas $(0.20$ $\mathrm{s})$ is longer than the decay of ripe bananas $(0.09 \mathrm{~s})$. This is observed elsewhere and is related to the large difference between the consistencies of bananas in these two conditions. ${ }^{22}$

This difference between ripe and unripe bananas was also observed in CWFP and CP-CWFP signals (Figure 7D). The variation in the amplitude of $T^{*}$ decay and $\left|\mathrm{M}_{\mathrm{ss}}\right|(0.40$ and 0.24 , respectively) indicates that $T_{1}>T_{2}$ in ripe bananas and that $T_{1} \gg T_{2}$ in unripe bananas.

Similar results are also observed for processed food products. Figure 8 shows the CPMG, CWFP and CP-CWFP signals for hotdog sausages (two brands) (Figures 8A and B) and regular and light mayonnaises (Figures $8 \mathrm{C}$ and D). The CPMG, CWFP and CP-CWFP signals (Figures 8A and B) of the two hotdog sausages were similar. The duration of the CPMG signal is longer for brand I sausage $(0.062 \mathrm{~s})$ than brand II $(0.056 \mathrm{~s})$, indicating a minimal difference in the composition of the two sausages. The $\mathrm{T}^{*}$ and $\left|\mathrm{M}_{\mathrm{ss}}\right|$ amplitude $(0.16)$ of the two sausages indicate that $\mathrm{T}_{1}>\mathrm{T}_{2}$.

Figures $8 \mathrm{C}$ and D depict the CPMG, CWFP and CP-CWFP signals of the regular (33\% fat) and light (24\% fat) mayonnaise samples from the same brand. Although the fat content in the light mayonnaise is $30 \%$ lower, the duration of the CPMG signal is only slightly longer $(0.15$ $\mathrm{s})$ than in regular mayonnaise $(0.14 \mathrm{~s}){ }^{7}$ However, the $\mathrm{T}^{*}$ and $\mathrm{M}_{\mathrm{ss}}$ of CWFP and CP-CWFP analyses are more sensitive to the fat content in mayonnaise. The $\left|\mathrm{M}_{\mathrm{ss}}\right|$ of regular mayonnaise is lower (0.22) than the light mayonnaise (0.28), thus, reflecting the difference in fat content. It is possible to process the full CWFP and CP-CWFP data using multivariate analysis; this would provide more information about the products and improved calibration and classification models compared to univariate analysis. ${ }^{5,7,20}$ 


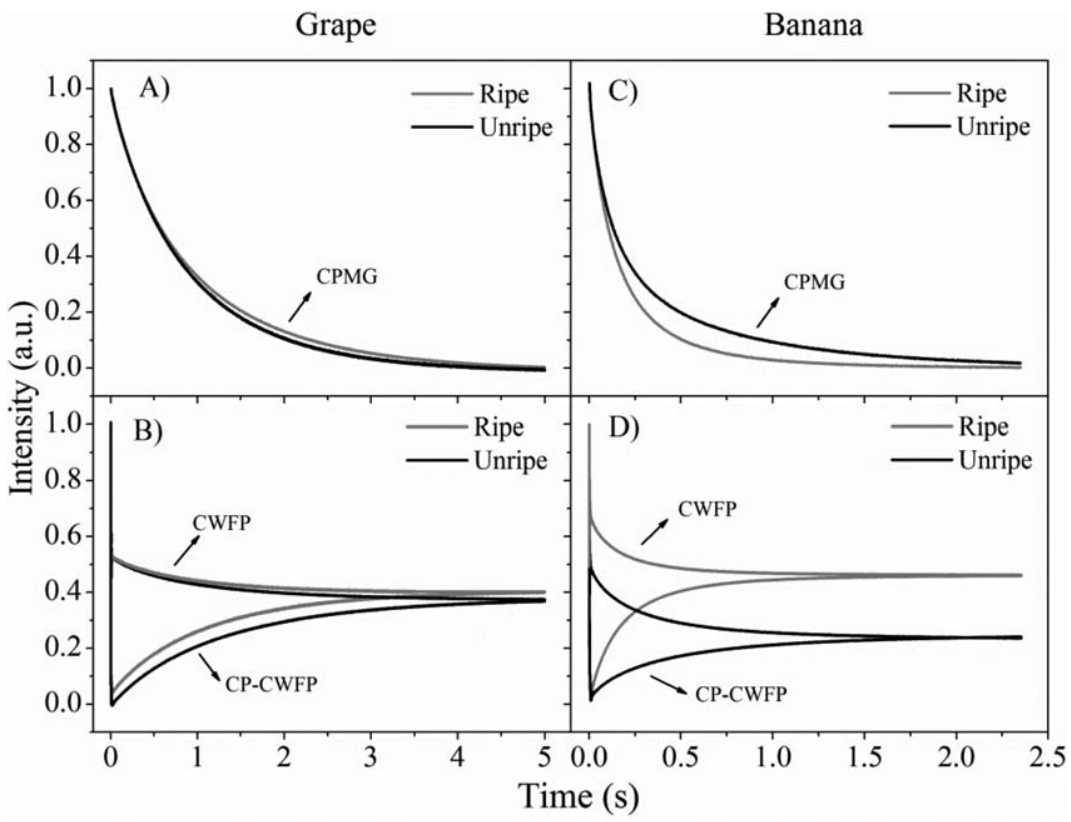

Figure 7 NMR signals intensities obtained for ripe and unripe grapes (A and $B)$ and bananas (C and D) using CPMG, CWFP and CP-CWFP pulse sequences.

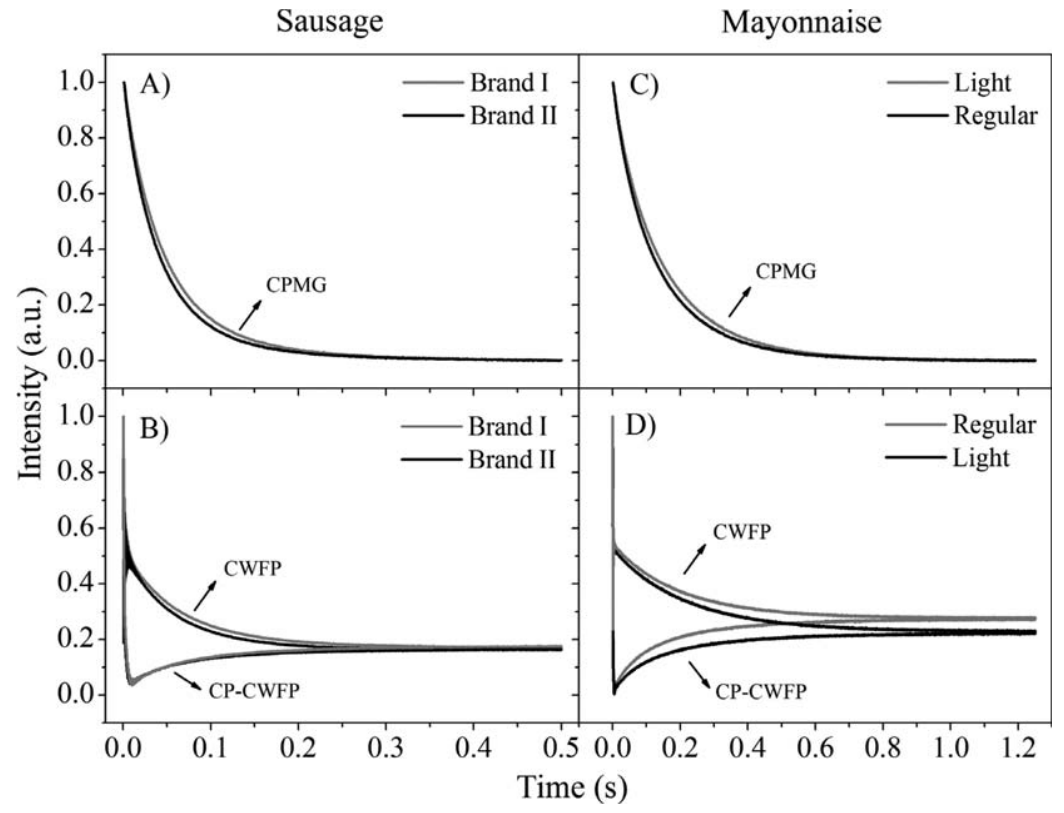

Figure 8 NMR signal intensities obtained from two brands of hotdog sausages (A and B), and regular and light mayonnaise $(C$ and $D)$ using CPMG, CWFP and CP-CWFP pulse sequences. 


\section{CONCLUSION}

Given the results presented, we conclude that the SSFP pulse sequences, using CWFP and/or CP-CWFP regimes, are alternatives and/or complementary methods to CPMG. The SSFP sequences are more efficient than CPMG for samples in which the change in $T_{1}$ is greater than $\mathrm{T}_{2}$. Furthermore, these sequences can be implemented in modern TD-NMR spectrometers.

\section{Acknowledgements}

This work was supported by FAPESP (process \# 2011/11160-3, 2012/20247-8, 2013/03770-1 and 2011/14099-3) and CNPq (Brazilian agencies). We are grateful to Professor Eduardo Ribeiro de Azevedo (IFSC- University of São Paulo) and Dr. Márcio Fernando Cobo (Bruker BioSpin), for the development of CWFP and CP-CWFP pulse sequences for minispec.

\section{References}

1 L.A. Colnago, R.B.V. Azeredo, A. Marchi-Netto, F.D. Andrade, T. Venâncio, Magn. Reson. Chem., 2011, 49, S113.

2 J. Van Duynhoven, A. Voda, M. Witek, H. Van As, Annu. Rep. NMR Spectrosc., 2010, 69, 145.

3 F. Dalitz, M. Cudaj, M. Maiwald, G. Guthausen, Prog. Nucl. Magn. Reson. Spectrosc., 2012, 60, 52.

4 F.D. de Andrade, A. Marchi-Netto, L.A. Colnago, Talanta, 2011, 84, 84.

5 F.M.V. Pereira, A.P. Rebellato, J.A.L. Pallone, L.A. Colnago, Food control, 2014 (in press),_DOI:10.1016/j.foodcont.2014.02.028

6 L.A. Colnago, F.D. Andrade, A.A. Souza, R.B.V. Azeredo, A.A. Lima, L.M. Cerioni, T. M. Osán, D.J. Pusiol, Chem. Eng. Technol., 2014, 37,191.

7 R.B.V. Azeredo, L.A. Colnago, M. Engelsberg, Anal. Chem., 2000, 72, 2401.

8 R.B.V. Azeredo, L.A. Colnago, A.A. Souza, M. Engelsberg, Anal. Chim. Acta, 2003, 478, 313.

9 R.B.V. Azeredo, M. Engelsberg, L.A. Colnago, Phys. Rev. E, 2001, 64, 16309.

10 L.A. Colnago, M. Engelsberg, A.A. Souza, L.L. Barbosa, Anal. Chem., 2007, 79, 1271.

11 T. Venâncio, M. Engelsberg, R.B.V. Azeredo, N.E.R. Alem, L.A. Colnago, J. Magn. Reson., 2005, 173, 34.

12 H.Y. Carr, Phys. Rev., 1958, 112, 1693.

13 R.R. Ernst, W.A. Anderson, Rev. Sci. Instrum., 1966, 37, 93.

14 A. Schwenk, Prog. NMR Spectrosc., 1985, 17, 69.

15 MATLAB; version 7.10.0 (R2010a), MathWorks Inc., Natick, Massachusetts, EUA, 2010.

16 P. Shkarin, R. G. S. Spencer, Concepts Magn. Reson., 1996, 8, 253.

17 J. Kronenbitter, A. Schwenk, J. Magn. Reson., 1977, 25, 147.

18 C.C. Correa, L.A. Forato, L.A. Colnago, Anal. Bioanal. Chem., 2009, 393, 1357.

20 F.M.V. Pereira, S.B. Pflanzer, T. Gomig, C.L. Gomes, P.E. Felício, L.A. Colnago, Talanta, 2013a, 108, 88.

20 P.M. Santos, C.C. Correa, L.A. Forato, R.R. Tullio, G.M. Cruz, L.A. Colnago, Food control, 2014, 38, 204.

21 F.M.V. Pereira, A.S. Carvalho, L.F. Cabeça, L.A. Colnago, Microchem. J., 2013b, 108, 14. 
22 F.Z. Ribeiro, L.V. Marconcini, I. B. Toledo, R.B.V. Azeredo, L.L. Barbosa, L.A. Colnago, J. Sci. Food Agric., 2010, 90, 2052.

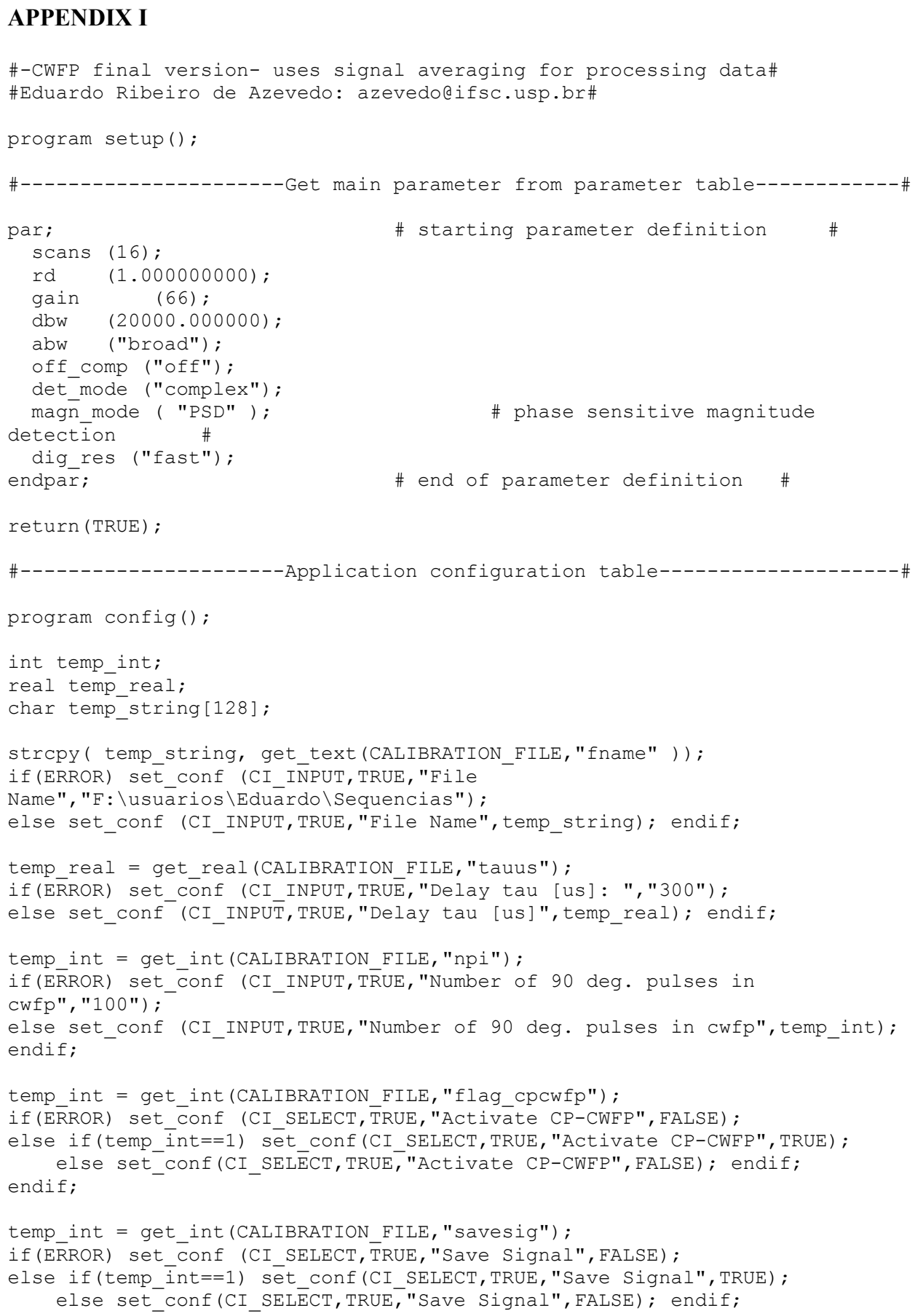


endif ;

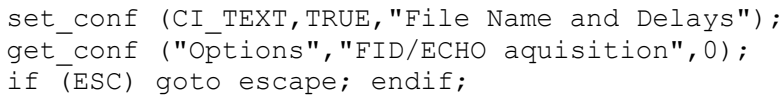




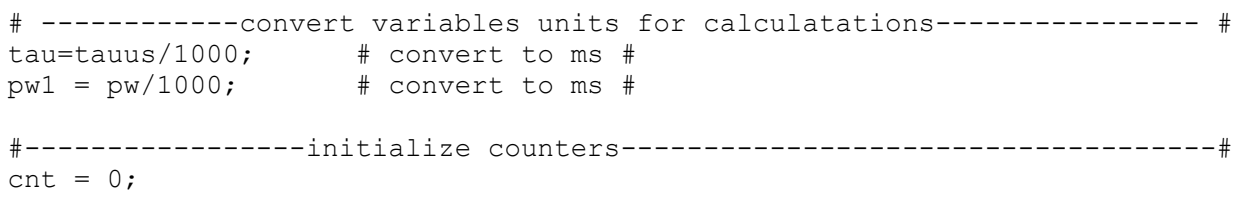

pulses;

sd $\left(1000 e^{-3)}\right.$; \# first delay for minimum time durantion of the sequence \#

cta;

if ( $f$ lag_cpcwfp==1)

ssp ( pw, ph90cp); \#90 degree pulse for CPCWFP \#

sd (rdt);

asd (acq, phrc); \# acq/2 delay is added to

compensate for an extra acq/2 delay in the first pulse of the cwfp loop \# sd ( tau/2.0 - pw1/2. - rdt - acq ); else

$\operatorname{ssp}(\mathrm{pw}, \mathrm{ph} 90)$;

\# 90 degree pulse for CWFP \#

sd (rdt);

asd (acq, phrc);

\# data acquisition \# 
sd ( tau - pw1/2. - rdt - acq );

\# acq/2 delay is added to compensate for an extra acq/2 delay in the first pulse of the cwfp loop \#

endif

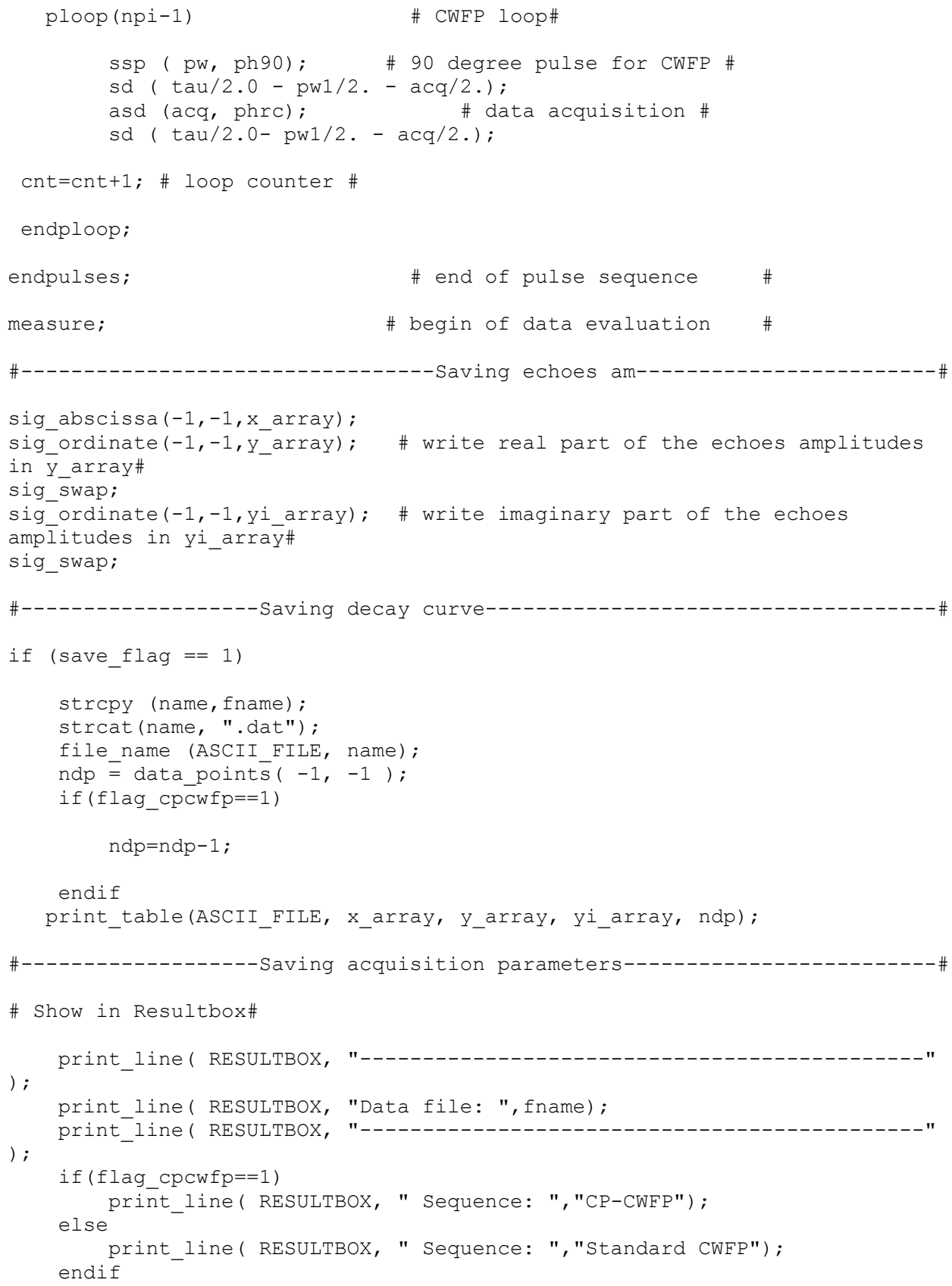


endif

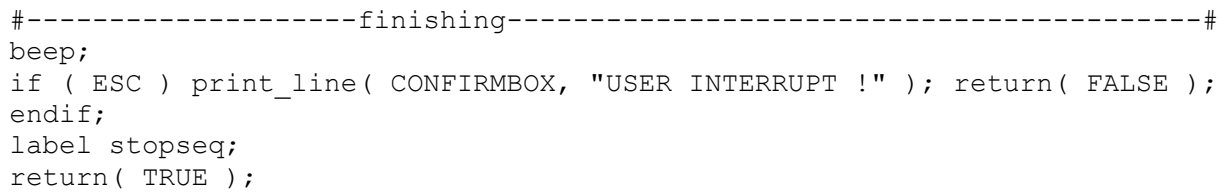

